DOI: $10.2478 / \mathrm{v} 10122-011-0001-\mathrm{x}$

\author{
A RT ICLES
}

\title{
SWAHILI: A DONOR LANGUAGE ${ }^{1}$
}

\author{
SERGIO BALDI
}

\begin{abstract}
Sergio Baldi. Swahili: A Donor Language. Lingua Posnaniensis, vol. LIII (1)/2011. The Poznań Society for the Advancement of the Arts and Sciences. PL ISSN 0079-4740, ISBN 978-83-7654-140-2, pp. 7-24.

Swahili is a Bantu language, more specifically a member of the Sabaki subgroup of North East Coast Bantu. Swahili was first written in Arabic script, in which there are manuscripts dating back to the early 18th century. A Roman alphabet which is now standard was introduced during the colonial period, although some Swahili-speaking Muslims continue to use Arabic script, especially in private correspondence. The cultural importance of Islam is reflected in the large number of loanwords from Arabic. Swahili is the national language in Tanzania and Kenya and largely spoken in the nearby countries. It has also an important role as a donor language in East Africa and Central Africa, not only to other Bantu idioms, but also to other languages belonging to different linguistic families.
\end{abstract}

Sergio Baldi, Università degli Studi di Napoli: “L’Orientale”, sbaldi@iuo.it

Swahili is one of the most studied African languages, as we can see from its very rich bibliographies (van Spaandonck 1965; Mioni 1967; Boucneau 1987; Baldi 1991). Many studies have been published on all aspects of the language, some of them dealing with Arabic influence and loans. But one aspect, which has not been considered up to now, is the role of Swahili as a donor language in East and Central Africa, not only to other Bantu idioms, but also to other neighbouring languages belonging to different linguistic families.

This paper is intended to investigate the spread of these Swahili loans in neighbouring languages, which got so many loans from Swahili. The survey is based on the analysis of words from some Bantu languages such as: Gikuyu, Lingala, Luganda, Nyakyusa, Shona as well as from languages belonging to different linguistic groups: Acholi, Bari, Madi, Pokot, Rendille, etc.

In the list given here, there are some Swahili words, which were lent to some east African languages. Most of these words were loans, mainly from Arabic, via Swahili. These items coming from my data base are collected from dictionaries and some other material, I came in contact with during my researches. The work, considering the enormous amount of work in progress in the field of African languages, is just a preliminary inquiry in topics not so well investigated.

${ }^{1}$ I would like to thank Mrs Fatuma Tandika, lettrice for Swahili at the Università degli Studi di Napoli "L'Orientale", for help and advice. Swahili words followed by an * are given by her and not found in consulted dictionaries. 
Here are some examples:

Swahili alhamisi [< Ar. kamīs (WeHR 262a) 'Thursday'] 'Thursday' > Kuria Aramisi 'Thursday';

Swahili almasi [< Ar. almās (WEHR 24a) 'diamond'] 'a diamond' > Nyakyusa alumasí 'diamond';

Swahili askari [< Ar. 'askarī (WeHR 613a) 'military, army (in compounds)'] 'soldier, guard, armed attendant' > Gikuyu thigari 'soldiery, police; soldier, policeman; companionship, consorting together'; Madi àsćkērè/àsìkárì 'soldier'; Pokot sìrkáalìyón 'soldier'; Rendille iskár 'soldier(s); police; gamewarden(s). Refers to any uniformed officer(s) in a position of authority';

Swahili askofu [< Ar. usquf (WEHR 17a) 'bishop'] 'a bishop' > Nyakyusa askofú 'bishop';

Swahili -badilisha [<Ar. badīl (WeHR 46b) 'substitute, alternate'] v. 'to cause to change; to get changed'> Gikuyu bandürithia v. 'to change, to alter, to get sth. changed, altered';

Swahili bafta [< Ar. bafta (WeHr 68a) 'calico, Indian cotton cloth'] 'a kind of thin bleached calico, used for lining garments'> Luganda bafùta 'calico';

Swahili bahari [<Ar. baḥr (WeHR 42b) 'sea'] 'sea, ocean'> Shona bahari 'sea';

Swahili bahasha ${ }^{2}$ [< Tur.] '(1) an envelope (2) satchel, bag'> Madi bàásà 'envelope';

Swahili baiyologi*/biolojia [< En.] 'biology'> Dholuo baiyoloji 'biology';

Swahili bakora [<? Ar. baqqāriy (FREYTAG: I, 142a) 'massif et solide (bâton), comme celui des bouviers'] 'a walking-stick, formerly one with a crooked handle' > Gikuyu mbokora 'walking-stick';

Swahili bakshishi [< Ar. baǩšǐs 'tip, gratuity' (WEHR 43b)] 'gratuity, gift, dole, tip' > Gikuyu mabacici 'largess, free gift, sth. added over and above a transaction'; Madi bàkàsísi 'tip; reward';

Swahili bakuli [< Ar. bāqūl (Dozy: I, 104b) 'cruche de terre poreuse pour l'eau'] 'basin, any large deep dish'> Luganda 'bbàkuli 'basin'; Madi bàkúli 'bowl'; Nyakyusa ibakúli 'bowl'; Pokot bàkúli 'bowl';

Swahili bamia ${ }^{4}$ [<Ar. bāmiya (WeHR 40a) 'gumbo, okra (Hibiscus esculentus L.)'] 'okra, lady's fingers (Abelmoschus esculentus)'> Nyakyusa ibamía 'okra, lady's finger';

Swahili barafu [< Pers. baraf] 'ice'> Rendille baraáfo 'ice, snow';

Swahili baraza [<Ar. barāza (KAZIMIRSKI: I, 110a) 'champ, vaste plaine sans arbres'] 'place of public audience or reception'> Gikuyu mbaratha 'public meeting, council, audience (held by a Government official)'; Lingala baraza/barza 'véranda';

Swahili baridi [< Ar. bārid (WEHR 51b) 'cold; cool'] 'cold, chill; wind, air' > ? Gikuyu mũbariti 'large tree (Grevillea robusta, gives deep shade)';

Swahili barua [<Ar. barwa (WEHR 56a) 'waste, scrap'] 'letter'> Gikuyu marua 'letter'; Luganda 'bbàluwà 'letter; note'; Madi bàrúà 'letter';

2 The word is marked as a loan from Turkish by JoHnson (1939: 24b).

3 This loan could have arrived directly from English.

4 Johnson (1939: 26b) suggests also a possible Persian origin. 
Swahili baruti ${ }^{5}$ [<Ar. bārūd (WeHR 39a) 'saltpeter; gunpowder'] 'gunpowder' > Gikuyu mbaruti 'gunpowder'; Lingala balúti 'poudre noire explosive, plomb à fusil'; Nyakyusa ibaluti' 'gunpowder';

Swahili basi $\left[<\right.$ En.] 'bus'> Dholuo bac ${ }^{6}$ 'bus';

Swahili basi ${ }^{7}$ [<Ar. bas (Roth-LaLy 50b) 'seulement; cela suffit'] conj. '(1) well (2) it is enough'> Madi básì adv. 'okay; fine; that's enough';

Swahili bastola ${ }^{8}$ [< Port.] 'a pistol' > Luganda bàsitoòla 'pistol';

Swahili bei [<Ar. baic (WeHR 86b) 'sale'] 'price'> Dholuo bei/beyi 'price';

Swahili bikira [< Ar. bikr (WEHR 70a) 'first-born; virgin'] 'a virgin'> Acooli bikira 'virgin'; Luganda mùbiikira 'virgin, nun', mùnuubiikira 'nun';

Swahili bilauri [< Ar. ballūr, billaur (WEHR 75b) 'crystal; crystal glass, glass'] 'glass, crystal; tumbler, wineglass' > Gikuyu mũraũni, mũraũri 'metal tumbler for drinking purposes'; Luganda kirawuli 'lamp glass; tumbler';

Swahili bilingani [<Ar. bādinjān/baidinjān (WEHR 38b) 'aubergine'] 'a dark purple vegetable, fruit of the egg plant, bringal, aubergine'> Gikuyu mbiringanya 'egg-plant, aubergine, brinjal'; Nyakyusa ibílínganía 'egg-plant, aubergine';

Swahili binzari/bizari [< Ar. bizr (WEHR 57a) 'spice'] 'small seed such as pepper'> Gikuyu bitharĩ/bitharĩ 'curry powder'; Madi bìnjárì 'curry powder';

Swahili birika [< Ar. ibrīq (WEHR 2a) 'pitcher, jug'] 'kettle' > Acooli biìnikà 'teapot'; Dholuo birika/binika 'kettle, teapot' ; Nyakyusa ilibilika 'kettle, teapot, jug, reservoir, cistern, tank';

Swahili blanketi/blangeti $[<$ En.] 'blanket' > Dholuo blayket 'blanket'; Madi bàlànggíti 'blanket';

Swahili boi $[<$ En.] 'a house servant' > Dholuo boyi'10 'a houseboy';

Swahili brigedia [< En.] 'brigadier' > Dholuo birigedia'11 'brigadier';

Swahili buku [<En.] 'book'> Dholuo buk'12 'book';

Swahili bunduki ${ }^{13}$ [<Ar. bunduqīya (WEHR 77a) 'gun' ] 'gun, rifle, musket' > Gikuyu bundũki, bindũki 'rifle, gun'; Luganda 'mmûndu 'gun, rifle';

5 JoHnson (1939: 20b) suggests a Turkish origin.

6 This loan could have arrived directly from English.

7 Johnson (1939: 31a) suggests also a possible Persian origin.

8 I think SACLEUX' (1939: 98a) etymology from Portuguese is more reliable than that one given by JoHNSON (1939: 31a): "? the same word through Arabic").

9 This loan could have arrived directly from English.

${ }_{10}$ This loan could have arrived directly from English.

11 This loan could have arrived directly from English.

12 This loan could have arrived directly from English.

${ }^{13}$ KNAPPERT (1972-1973: 293, note 18) affirme: "La forme hausa bindiga vient d'un terme de la Lingua franca désignant le fusil venediga; ce mot, en portugais ou en espagnol, est la forme adjectivale de Venise, ancien port de transbordement d'armes à feu". Le mot en luganda est un emprunt au swahili bunduki, "itself a loan from Turkish via Arabic. The origin of this word is the Greek pontikon (hazelnut), referring to the shape of a musket bullet. The Ganda form of the word can be explained by the "law of Meinhof", which states that the first of two consecutive voiced pre-nasalized plosive consonants must become a nasal. The loss of the last syllable is already found in Luo bunde; the Nilotic languages prefer words of one or two syllables" (KNAPPERT 1999: 209). 
Swahili bure a./adv. 'useless; free; empty'> Dholuo bure adv. 'for nothing';

Swahili bushuti [? < Arabic basāt ${ }^{14}$ 'covering'] 'a long cloak worn by some men, decorated with goldd threadwork' > Luganda bùsuuti 'Arab robe used by certain chiefs on ceremonial occasions';

Swahili buti [< En.] 'boot; trunk'> Dholuo buut '15 'carvas shoes';

Swahili chafu a. 'dirty' > Rendille chaáfo 'dirt';

Swahili chai $^{16}$ [< Ar. šây (WeHr 451a) 'tea'] 'tea' > Nyakyusa ikyai 'tea'; Rendille cháay 'tea';

Swahili cherehani [< Pers.] 'sewing machine' > Acooli càráàn 'sewing machine'; Dholuo caran 'sewing machine';

Swahili choo 'water-closet, cess-pit'> Rendille chóow 'latrine, toilet (usually refers to an outside pit latrine)';

Swahili chooni 'in the water-closet' > Kuria ichorooni 'latrine, lavatory'; Madi sòrónì 'excrement; latrine, lavatory';

Swahili chotara ${ }^{17}$ [? < Ar.] 'a half-breed person'> Gikuyu cotara 'half-caste';

Swahili chungwa, pl. machungwa 'orange'> Rendille machuúnga 'orange (fruit)';

Swahili chupa [ ? < Ar.] 'a bottle'> Acooli cupà 'bottle'; Ateso ecupa 'bottle'; Dholuo cupa 'bottle'; Rendille chúbba 'bottle';

Swahili dakika [<Ar. daqīqa (WeHr 288a) 'minute (time unit)'] 'a minute, the 60th part of an hour, but often used as any small division of time' > Gikuyu ndagîka, ndakinga 'a minute'; Luganda 'ddakiika 'minute'; Madi dákt́kà 'minute; one minute; a moment'; Rendille dekeéka 'minute';

Swahili daktari [< Ar. duktūr, pl. dakātira (WEHR 288b) 'doctor'] 'doctor of medicine' $>$ Madi dàkìtárì 'medical personnel (doctors, nurses etc.); hospital; health centre, etc.'; Rendille ilkitárri 'doctor';

Swahili daraja [< Ar. daraja (WEHR 277a) 'rank'] 'step, set of steps, stairs, staircase, bridge; degree, rank, dignity, social station' > Gikuyu ndaraca 'bridge'; Pokot tàrácà 'bridge'; Rendille daraánja/daraáncha 'bridge; culvert (conduit for water pipe or drain under a road)';

Swahili dawa [< Ar. dawā' (WeHr 304b) 'remedy, medicament'] 'medicine, medicament ; charm, talisman' > Dholuo dawa 'medicine'; Gikuyu ndawa 'medicine, cure'; Rendille ildáwa 'medicine, ointment; treatment (for illness)';

Swahili debe [<Hind.] 'tin can, commonly of 4 gallons, such as those in which petrol and kerosene is imported and sold' > Acooli debe 'a metal vessel, tin can, pail'; Dholuo debe 'tin can, pail, bucket'; Madi dé 'bè/ndé 'bè 'a tin of about four gallons in size';

Swahili dhahabu [< Ar. dahab (WeHR 313b) 'gold'] 'gold' > Acooli dab 'gold'; Bari dakap 'gold'; Dholuo dhahabu 'gold'; Gikuyu thahabu 'gold'; Luganda zaabù 'gold'; Madi dá 'bù 'gold'; Nyakyusa sahabu 'gold'; Rendille 'dáhab 'gold';

${ }^{14}$ It is Johnson's hypothesis (1939: 43a).

15 This loan could have arrived directly from English.

${ }^{16}$ In Swahili the word according to JoHnson (1939: 47b) is from Persian or Hindi.

${ }_{17}$ In Swahili the word itself could be a loan from Arabic šatr (WEHR 1966: 471b) 'partition, division', according to Johnson (1939: 61a), but according to SACLEUX (1939: 151a) it is from Hindoustani. 
Swahili dini [< Ar. dīn (WEHR 306a) 'religion'] 'religion, creed, worship' > Dholuo dini 'religion'; Gikuyu ndini 'religion'; Kuria idiini 'a religion, religious instructions'; Luganda 'ddiìni 'religion; creed'; Madi díni 'religion';

Swahili dira [< Ar. dā'ira (WeHR 301a) 'circle; compass'] 'a mariner's compass' > Luganda 'ddiirâ 'compass';

Swahili $d u b u$ [< Ar. dubb (WeHR 269b) 'bear'] 'bear' > Gikuyu nduba 'ours'; Luganda 'ddubù 'ours';

Swahili duka [<Ar. dukkān (WEHR 288b) 'bench; store, shop'] 'shop, stall'> Acooli dokà, dvkan 'merchant's shop'; Dholuo duka 'merchant shop'; Gikuyu nduka, matuka 'shop'; Madi dìkánì 'shop'; Pokot 'dúkà 'shop'; Rendille iltukáan/ildukáan 'shop; store; trading post';

Swahili elfu [< Ar. alf (WeHr 23a) 'thousand'] n./a. 'a thousand' > Dholuo alip 'thousand'; Gikuyu warubu 'thousand'; Luena élefu 'thousand'; Rendille álif/élif 'thousand';

Swahili faida [< Ar. fä'ida (WEHR 735a) 'utility, benefit, profit, interest'] 'profit, gain, advantage, interest' > Gikuyu baita 'profit';

Swahili -faransa [< Ar. faransā (WeHr 710b) 'France'] a. 'French' > Gikuyu Baranja 'France';

Swahili farasi [< Ar. faras (WeHR 704b) 'horse'] 'horse' > Gikuyu mbarathi 'horse'; Lingala farása 'horse'; Luganda 'mbalaàsi 'horse';

Swahili fataki [? < Arabic fatq (WEHR 695a) 'rip, cleft, fissure, slit, slash'] 'gun cap' > Lingala fatáki 'fusil à piston';

Swahili fedha [<Ar. fidda (WEHR 717a) 'silver'] 'silver; money, coin, cash-in general' > Gikuyu betha 'silver'; Luganda 'ffeezà 'silver'; Nyakyusa fesa 'silver';

Swahili fereji [ $<$ Ar. farj (WEHR 702a) 'opening, aperture, gap, breach; pudendum oth female, vulva'] 'a large ditch, channel'> Gikuyu mũberethi 'pipe, pipeline, tube';

Swahili -fikiri [<Ar. fikr (WEHR 724b) 'thinking'] v. 'think (about), ponder (over), meditate (upon), consider, reflect (about)' > Gikuyu thikiri, bikiri 'thoughts, meditations, brooding';

Swahili fitina [< Ar. fitna (WEHR 696a) 'intrigue; sedition, riot, discord, dissension'] 'discord, variance, antagonism, quarrelling, misunderstanding, mischief' > Gikuyu bitina 'false allegation, spite, conspirancy against a person, deliberate mischief; underhand dealing, discord, antagonism, mischief'; Madi fítt̄nà 'envy, jealousy';

Swahili frasila ${ }^{18}$ [? < Ar.] 'a measure of weight, about 35 lb.' > Luganda fulààsira 'frasila, measure of weight, about 35 lb.';

Swahili fulana [< En.] 'flannel; vest; T-shirt; sweator' > Madi fàlànì, fùlànì, fènílà 'vest, flannel';

Swahili -funga v. 'to fasten, to tie'> Luena ku-fúnga v. 'fermer';

Swahili galoni* [< En.] 'gallon'> Acooli jolon 'gallon'; Bari jölun, jölunyön 'gallon'; Lotuxo ajvlvn 'gallon';

${ }^{18}$ In Swahili the word itself could be a loan from (?) Arabic faar and saah i.e. a full basket, or perh. corruption of Eng. parcel, the commercial term. 
Swahili gari 'any vehicle on wheels' > Acooli gaari 'car, cycle'; Bari gari 'bicycle'; Dholuo gari 'bicycle'; Rendille gaári 'vehicle (of any kind); car, lorry, truck, buses';

Swahili -geni a. 'strange, foreign'> Kuria -geni a. 'strange, foreign';

Swahili ghorofa/orofa [<Ar. gurfa (WEHR 670b) 'upstairs room, room on an upper floor'] 'upper story, upper room'> Gikuyu ngorba 'store; upper rooms'; Luganda 'ggòloòfa 'upper story, upstairs';

Swahili gunia [< Hind. kawn] 'a coarse bag or sack chiefly used for packing rice and grain, also the material from which it is made, sackcloth'> Acooli gùniià 'sack'; Bari guniya 'sack'; Madi gùtíà/gùníà 'a sack';

Swahili haki [<Ar. haqq (WeHr 192a) 'one's due; duty; right'] 'justice, right, lawfulness' $>$ Kuria ehaki 'justice, fairness; share, one's due';

Swahili haradali [< Ar. kardal (WeHR 234a) 'mustard seeds; mustard'] 'mustard' > Gikuyu karatarĩ '(Bibl.) mustard seed';

Swahili hariri [< Ar. harīr (WEHR 165a) 'silk'] 'silk'> Luganda àliìri, liìri 'silk';

Swahili hatamu [<Ar. kaț (WEHR 248a) 'nose, muzzle (of an animal)'] 'bride'> Gikuyu matamu 'bit, bridle, and reins ';

Swahili hatari [<Ar. katar (WEHR 247a) 'danger, peril; risk'] 'danger, peril, risk'> Kuria -hataari a. 'dangerous';

Swahili haya! [< Ar. hayyā interj. (WeHr 1041a) 'up! come on! let's go!'] int. 'used to call to action or effort, Come on! Now then! Work away! Step out! Make haste!' > Gikuyu haya, heya int. 'expr. 1. surprise; there, didn't I tell you so? 2. encouragement; well done! come on!';

Swahili hema [< Ar. kaima (WEHR 269a) 'tent'] 'tent' > Gikuyu hema 'tent'; Lingala yéma 'tent'; Luena hèma 'tarpaulin'; Luganda weemà 'tent'; Rendille eéma 'tent';

Swahili hesabu, hisabu [< Ar. hisāb (WEHr 176a) 'arithmetic, reckoning'] 'reckoning; arithmetic'> Gikuyu ithabu 'sum, bill, account; arithmetic';

Swahili hirizi [<Ar. hirz (WEHr 167b) 'amulet'] 'charm, amulet'> Gikuyu gĩthitũ 'amulet'; Luganda èyirizì 'prayer-beads of Muslims; charm, amulet (often in the form of beads); phylacterries';

Swahili homa [<Ar. humma (WeHr 203b) 'fever'] 'fever, any sickness with a high temperature' > Gikuyu homa 'the common cold';

Swahili hukumu [< Ar. ḩukm (WEHR 196a) 'judgment, verdict; condemnation; power; government'] n./v. 'judgement; give an official pronouncement (on)' > Gikuyu ũkümũ 'sentence, verdict, judgement; insubordination; punishment, correction';

Swahili ijumaa [<Ar. jumca (WeHR 135a) 'Friday'] 'Friday'> Gikuyu njuma 'Saturday'; Kuria Ichumaa 'Friday';

Swahili Injili/Anjili [<Ar. injīl (WeHr 30a) 'gospel'] 'the New Testament'> Gikuyu injiri 'gospel'; Luganda 'Njirì 'Gospel';

Swahili jamhuri [<Ar. jumhūrīya (WEHR 138a) 'republic'] 'a meeting; a republic'> Kuria Chamuhuri 'Republic Day (national holiday)';

Swahili jani, pl. majani 'leaf, blade of grass' > Rendille majjaáni 'tea-leaves'; 
Swahili jela [< En.] 'jail' > Rendille jeéla 'jail';

Swahili jeshi [<Ar. jaiš (WEHR 150b) 'army, troops'] 'a great company, assemblage, host, troop, army' > Nyakyusa ilijesi 'army, military';

Swahili joho [< Ar. jū $\underline{k}$ (WEHR 146a) 'broadcloth'] 'woollen cloth'> Luganda ’jjòwô 'woollen material; flannel';

Swahili Jumapili 'Sunday' > Kuria Chumabiiri 'Sunday';

Swahili Jumatatu 'Monday' > Kuria Chumatatu 'Monday';

Swahili Jumamosi 'Saturday' > Kuria Chumamosi 'Saturday';

Swahili Jumanne 'Tuesday' > Kuria Chuma'inne 'Tuesday';

Swahili Jumatano 'Wednesay' > Kuria Chumataano 'Wednesay';

Swahili jumla [<Ar. jumla (WeHr 137a) 'totality'] 'sum, total, a lot' > Gikuyu njamura 'wholesale; all together';

Swahili juzi 'the day before yesterday'> Luganda ’jjuuzi adv. 'the day before yesterday; the other day';

Swahili kabati [< En. cupboard] 'a cupboard, sideboard'> Kuria ekaabaati 'a cupboard'; Luena kabádi 'garde-manger';

Swahili kabila [< Ar. qabīla (WEHR 741a) 'tribe'] 'tribe, clan'> Gikuyu kabira 'sort, kind, breed';

Swahili kaburi [<Ar. qabr (WEHR 738a) 'tomb'] 'grave, tomb'> Dholuo kaburini 'cemetery'; Gikuyu kaburi 'graveyard';

Swahili kahawa [< Ar. qahwa (WeHr 795a) 'coffee'] 'coffee'> Gikuyu kahũa 'coffee; coffee plants'; Luganda kaawà 'coffee'; Nyakyusa ikahabwa 'coffee'; Pokot káawèn 'coffee'; Rendille gaáwo 'coffee';

Swahili kalamu [<Ar. qalam ${ }^{19}$ (WEHR 788a) 'pen'] 'pen or pencil' > Gikuyu karamu 'pen, pencil'; Kuria ekaraamu 'a pen or pencil';

Swahili kamba [? < Ar. kinbār (KAZIMIRSKI: II, 933b) 'corde faite en fibres de cocotier'] 'cord, rope (the most generic term)' > Gikuyu ikamba, kamba 'sisal tow; rope, cord';

Swahili kanisa [<Ar. kanīsa (WEHR 842b) 'church'] 'church'> Kuria ekanisa 'a church'; Luganda 'kkànisà 'church'; Pokot kànísà 'church'; Rendille kanissa 'church';

Swahili karani [<Ar. qara'a (WEHR 753a) v. 'to recite; to read'] 'clerk, secretary' > Acooli kàráàn 'clerk'; Bari kawurönit, kawurök 'clerk'; Luganda kalàani 'clerk';

Swahili karatasi [< Ar. qirțās (WEHR 757b) 'paper'] 'paper, a piece of paper' > Dholuo karatac 'a sheet of paper'; Gikuyu karatathi 'paper'; Kuria ekarataasi, iritarataasi 'paper, a piece of paper'; Kuria ekarata 'a playing card';

Swahili karibu [<Ar. qarīb (WeHr 754b) 'near (in place and time), nearby, close at hand; relative, relation'] n./adv./prep. 'near relation, kinsman; near, close to; presently, shortly; nearly, almost, about' > Luganda kàribù int. 'Come in!';

Swahili kasisi [< Ar. qissīs (WEHR 762a) 'priest, minister, pastor'] 'priest' > Gikuyu mũgathithi 'priest, presbyter';

19 The Arabic word itself is a loan from Greek $\kappa \alpha \dot{\lambda} \alpha \mu o \varsigma$ 'roseau; calame en roseau, stylet', cf. latin calamus 'roseau' (BALDI 2008: 414, no. 2335). 
Swahili kataa [<Ar. qiț a (WEHR 776b) 'piece, fragment; part'] 'a cutting, piece, part, portion, section, fraction'> ? Gikuyu gata v. 'cut';

Swahili katani [<Ar. kattān (kittān) (WEHR 815a) 'linen'] 'hemp, sisal fibre (Agave sisalana)'> Gikuyu gatani 'flax, linen';

Swahili kazi 'work'> Luena kâshi 'work'; Rendille ilkaássi '1. work, employment 2. a specific task, job';

Swahili kiasi 'the charge of a gun, cartridge'> Luganda èkyâsi 'cartridge';

Swahili kiberiti, kibiriti [<Ar. kibrīt (WEHR 811a) 'sulphur; matches'] 'sulphur; a match' $>$ Luganda kibiriiti 'sulphur; match'; Madi kibiritìtibiritì 'matches; matchbox'; Pokot kipiríitò 'match';

Swahili kiboko 'whip' > Acooli kibögò 'whip of hippo skin'; Dholuo kiboko 'a whip made from hippo hide';

Swahili kijiko, dim. of mwiko 'a spoon, or instrument resembling' > Dholuo kijiko 'a spoon'; Rendille kucchúga 'spoon';

Swahili kikebe/mkebe (SACLEUX 1939: 560b) 'boîte ronde avec couvercle en calotte, en métal précieux'> Acooli gikebè 'small tin box (as for tobacco)';

Swahili kikombe 'a vessel scraped or hollowed out, and so dim. of kombe, a small dish, cup, basin, mug'> Rendille kokkóob/okkóob/kóob 'cup; mug';

Swahili kilabu [<En.] 'club'> Madi kùlúbù 'hotel';

Swahili kitabu [<Ar. kitāb, pl. kutub (WeHR 812b) 'book'] 'book'> Gikuyu gĩtabu 'book; pamplet, exercise- or notebook, magazine'; Luganda kitabo 'book'; Madi kìtá 'bù 'book'; Nyakyusa ikitabu 'book'; Pokot kitapu 'book';

Swahili kodi $i^{20}$ [? < Ar.] 'rent, tax'> Rendille koóddi 'tax';

Swahili kofia [< Ar. kūfìya (WeHR 846b) 'kaffiyeh'] 'cap, usu. a fez'> Gikuyu ngũbia, ngobia 'cap, hat; tilde, diacritical mark over vowel';

Swahili -koroga v. 'to stir, to mix by stirring' > Rendille koróga 'powdered milk';

Swahili koti [<En. coat] 'a coat, a jacket'> Luena kòtshi 'veston';

Swahili kura [<Ar. qara'a v. (WeHR 757b) 'to knock' VIII 'to vote'] 'a lot, i.e. in casting lots'> Gikuyu kura 'vote'; Rendille kúra 'election';

Swahili labda [<Ar. lā budda (WeHr 44a) 'it is necessary'] adv. 'perhaps, it seems so, no doubt, probably, possibly'> Acooli búlê adv. 'perhaps, possibly; nearly';

Swahili laghai a./v./n., ragai, mlaghai [<Ar. rāga (WEHR 368a) 'to turn off' (III) rāwa $\bar{g} a$ 'to deal in an underhanded, fraudulent manner, to dodge'] 'deceitful, false, sly, dishonest' $>$ Gikuyu raghaĩ 'good-for-nothing, wastrel, scoundrel; (of a child) troublesome rascal; rubbish, worthless thing';

Swahili lami 'pitch, tar'> Gikuyu rami 'pitch, tar, and sometimes used for any dark viscous stuff';

Swahili lazima [< Ar. lazima v. (WeHR 864b) 'to attend; to be imperative'] 'necessity, obligation, surety, bail, responsibility'> Gikuyu rathima, in wĩra wa 'compulsory labour';

${ }^{20}$ In Swahili the word itself could be a loan from? Hind. kar 'tax or fee' or from Pers. kuud 'stack or heap of corn, i.e. as a tithe' according to JoHNSON (1939: 215a), but I prefer SACLEUX' hypothesis (1939: 429a). 
Swahili lozi [<Ar. lauz (WEHR 883a) 'almond(s)'] 'an almond, fruit of the mlozi' > Gikuyu njothi 'nut, almond';

Swahili lulu [<Ar. lu'lu' (WeHr 852b) 'pearls'] 'pearl'> Luganda luùlu, èruùlu 'pearl';

Swahili mafuta 'oil, fat, grease (of any kind)' > Luganda àmàfutà 'oil; petrol; paraffin; butter';

Swahili mahabusi [<Ar. maḥbūs (WeHR 154a) 'imprisoned; prisoner'] 'a prisoner awaiting trial, and the place where such prisoners are kept' > Gikuyu mũmabuthu, mabuthu 'prisoner, convict, the accused';

Swahili malaika [< Ar. mal'ak, pl. malā'ika (WEHR 922b) 'angel'] 'a messenger, an angel, a good spirit' > Ateso emalaika 'angel'; Dholuo malaika, malaike 'angel'; Gikuyu mũraika 'angel'; Luganda màlayika 'angel'; Madi màlát̄kà 'angel';

Swahili malaya [< Ar. malīya, pl. malāyā (WEHR 924a) '(tun.) garment of Bedouin women'] 'a prostitute, either male or female' > Acooli màlayà 'harlot'; Dholuo malaya 'harlot, prostitute'; Luganda màlaàyà 'harlot; banana on Entebe peninsula'; Pokot cèmàláyán 'prostitute';

Swahili mali [<Ar. māl (WEHR 931b) 'goods; wealth; money; stock'] 'property, goods, wealth, riches, possession'> Luganda 'mmaàli 'possessions; money; wealth'; Shona mari 'money (cash)'; Xhosa imali ( ? via Swahili) 'money';

Swahili marashi pl. 'scent, liquid perfume'> Rendille mirícchi 'perfume';

Swahili maskini [<Ar. miskīn (WEHR 909b) 'poor'] 'a poor man, beggar' > Rendille miksín/miskiin 'poor person';

Swahili matana 'leprosy' > Luganda màtanà 'tuberculous leprosy';

Swahili mboga 'a large edible gourd'> Rendille imboóga '1. vegetables 2. stew (can contain meat as well as vegetables';

Swahili mchele 'rice'> Luena mutshèle 'riz';

Swahili mdalasini [< Ar. dārșiny (GHALEB: II, n 17 468) 'Cinnamomum zeylanicum'] 'the cinnamon tree, Cinnamomum zeylanicum' > Gikuyu mũtarathini, ndarathini 'cinnamon';

Swahili merikebu [< Ar. markab (WEHR 357a) 'ship, boat, vessel'] 'a ship, esp. of foreign construction, as contr. with the native vessel chombo'> Gikuyu merikebu, marikabu 'ship, steamer'; Luganda màlèkebù 'ship';

Swahili meza [< Port. mesa < Lat. mēnsa] 'table'> Acooli mecà 'table; court fee'; Dholuo meja 'table'; Gikuyu metha 'table'; Kuria emeesa 'a table'; Luganda 'mmeezà 'table'; Madi mézà 'table'; Nyakyusa imeesa 'table'; Pokot mesa 'table'; Somali miis 'table';

Swahili mguu 'the leg - of man or any kind of living creature'> Luena mukúlu 'jambe';

Swahili Mhindi 'a native of India'> Rendille Ilmoóndi/Moóndi 'Indian(s), Asian(s) (referring to Kenyans of Indian descent)';

Swahili mia [<Ar. mi'a, pl. mi'ūn, mi'āt (WEHR 889b) 'hundred'] n./a. 'hundred'> Acooli miía 'nut, almond'; Dholuo miya 'hundred'; Kuria mia 'one hundred'; Luena mía 'hundred';

Swahili Misri [<Ar. miṣr, (colloq.) mașr (WeHr 911b) 'Egypt; Cairo'] 'Egypt' > Luganda Mùmisiri 'Egyptian'; 
Swahili miwani [< Ar. mucāyana (WeHR 663b) 'view(ing), examination, survey(ing); inspection; surveillance'] 'a pair of spectacles, eye-glasses'> Gikuyu mĩwani 'spectacles, (eye)glasses';

Swahili mizani [< Ar. mīzān (WeHR 1065b) 'balance'] 'balance; scales; clock, watch'> Luganda mìzaàni 'scales for weighing; spring balance';

Swahili mjumbe 'messanger, deputed person, delegate' > Rendille Mujuúmbe 'Member of Parliament';

Swahili mkasi, makasi [<Ar. miqașs, pl. al-maqāṣs (WEHR 766a) 'scissors'] 'scissors'> Gikuyu magathĩ 'scissors, shears'; Lingala makási 'ciseaux, cisailles’; Luganda màkânsi 'scissors'; Pokot màkásò 'scissors';

Swahili mkate 'a loaf, cake; bread' > Acooli mùgàtì 'bread'; Ateso emugati 'bread'; Dholuo mugati 'bread'; Luena mukáti' ${ }^{21}$ 'pain'; Pokot mùkàtìyán 'bread'; Rendille makaáte 'bread; chapati (crisp pancake)';

Swahili mkebe [< Ar. kūb (WEHR 845a) 'drinking glass'] 'pot, can, canister, mug, a cigarette case' > Gikuyu mũkebe 'tin, tin-can';

Swahili mlimau ${ }^{22}[<$ Port. limão] 'the lemon tree (Citrus limonum)' > Luganda òmùlimaàwa 'lemon tree';

Swahili mpira 'a tree producing india-rubber; the substance india-rubber' > Acooli mùpirà 'flannel; india-rubber ball'; Nyakyusa ipila 'rubber'; Pokot mbìrà 'rubber, rubber ball'; Rendille imbira '1. rubber or plastic object; plastic wire. 2. plastic containers. 3. balls';

Swahili mpunga 'the rice plant, Oryza sativa'> Ateso emupuga 'rice';

Swahili msalaba [<Ar. șalīb, pl. șulbān, șulub (WeHR 521b) 'cross'] 'a cross, anything in the form of a cross' > Gikuyu mũtharaba 'cross (for crucifixion)';

Swahili mshahara [<Ar. mušāhara (WeHR 490b) 'monthly salary; pl. monthly payments, monthly allowances'] 'monthly wages, regular salary' > Gikuyu mũcara 'wages, salary'; Pokot misiárà 'payment, salary'; Rendille macchaára 'salary, wage';

Swahili mshihiri 'an Arab from Sheher in South Arabia, usu. engaged in manual trades and labour, fishing, \&c.'> Gikuyu mũcihiri 'Arab labourer from S. Arabia; half-breed';

Swahili mshumaa [< Ar. šamca, šamaca (WeHR 486b) '(wax) candles'] 'a candle' > Gikuyu mũcumaa 'candle';

Swahili msikiti [<Ar. masjid²3 (WeHR 397b) 'mosque'] 'a mosque'> Gikuyu mũthigitibarĩ 'church building; mosque'; Luganda mùzigiti 'mosque';

Swahili mstari [< Ar. mistara (WeHR 410a) 'ruler; underlines'] 'a line, a line ruled or marked, a row'> Gikuyu mũthitarĩ 'line, row'; Shona mutsara 'line (? via Swahili)';

${ }^{21}$ In Luena the loan has this particular meaning from the Swahili etymological sense of the word 'something cut'.

22 In Swahili the word itself is a loan from Persian and Hindustani limau according to Johnson (1939: 290b), but SACLEUX (1939: 572a) does not say anything more prudently.

23 BALDi (2008: 238) observes: “Dialectal algérien mesjed, berbère mauritanien ms'id lieu d'adoration, mosquée (Nicolas: 127) où l'on constate la réduction $\mathrm{du} / \mathrm{j} /$. En swahili, il y a l'influence de l'arabe égyptien, où /j/ > /g/". 
Swahili msumari [< Ar. mismār (WeHR 429b) 'nail'] 'nail, large pin, or anything similar in appearance or use' > Nyakyusa unsúmali 'nail'; Rendille ussumáar 'metal nail (used in carpentry)';

Swahili msumeno 'saw'> Madi mùsùménì 'saw, for cutting';

Swahili mwalimu [< Ar. mucallim (WEHR 637a) 'teacher'] 'a learned man, a teacher, a schoolmaster' > Rendille maalimo 'teacher';

Swahili mwarabu [< Ar. 'arab (WeHr 601a) 'Arab'] 'Arab' > Gikuyu Mwarabu 'an Arab'; Luganda Mùwalabù 'Arab'; Nyakyusa umwalabu 'Arab';

Swahili Mwislamu [<Ar. islām (WeHR 426a) 'the religion of Islam'] 'a Muhammadan'> Rendille Múslam 'Muslim';

Swahili Myunani [<Ar. al-yūnān (WeHR 1110b) 'the Greeks; Greece'] 'an ancient Greek' $>$ Luganda Mùyonaàni 'a Greek';

Swahili Mzungu 'European'> Gikuyu Mũthũngũ 'European'; Luganda -zûngu a. 'European';

Swahili nabii [< Ar. nabiy (WEHR 941b) 'prophet'] 'a prophet, a preacher of righteousness, one who foretells the future'> Gikuyu mũnabii 'prophet';

Swahili nanasi [< Pers.] 'pineapple' > Kuria enaanaasi 'a pineapple';

Swahili nauli [< Ar. naulūn, nāwulūn (WEHR 1012b) 'freightage, freight'] n./v. 'fare, charge for freight (or conveyance), passage money'> Nyakyusa inauli '(e.g. bus) fare';

Swahili ndege 'a bird' > Rendille indége 'aeroplane';

Swahili ndururu 24 'five cents'> Rendille in'duruúru 'Kenyan five-cent coin';

Swahili neno, pl. maneno 'a word, utterance'> Luena manéno 'discussion, dispute';

Swahili ngamia 'a camel' > Ateso egamira 'camel'; Gikuyu ngamĩra 'camel'; Luganda è yyàmirà 'camel';

Swahili nukta [< Ar. nuqta (WEHR 993a) 'point'] 'a dot, point, mark, spot, vowel sign; a second (of time)'> Luganda 'nnukùta 'letter of the alphabet';

Swahili nusu [<Ar. nișf, nușf(WEHR 971a) 'half'] 'a half, a part, a portion, a bit'> Gikuyu nuthu 'half; 50-cent piece; portion more or less than a half'; Kuria enoso 'a half'; Luganda 'nnusù 'half'; Rendille nyúus 'half';

Swahili nyundo 'a hammer'> Rendille nyuúndu 'hammer';

Swahili pamba 'cotton'> Acooli pamà 'cotton';

Swahili pesa [< Port. péso < Lat. pensum] 'a piece, the Indian quarter anna; money'; Gikuyu rübeca, mbeca '(formerly of Indian and East African coinage) pice (now reckoned as 3 cents, in N.K. 4 cents); (cl. 10 only) money in general'; Luganda 'ppeesà ${ }^{25}$ 'button; coin'; Madi pésà 'money';

24 See BAKHRESSA 1992: 282a.

${ }^{25}$ KNAPPERT (1999: 206) says: "The Luganda word èppeesà (a button) is derived from Swahili pesa (a coin, money) via Hindustani from Portuguese pesa. The ultimate origin of the word is again Latin, i.e. pensa (a weight) from pendere (to hang). That the word has travelled to East Africa via India is proved by its shape: if it had come directly from Portuguese, it would have been pronounced with a " $z$ " instead of " $s$ " - compare Swahili meza (table) via Portuguese mesa from Latin mensa. In Hindustani, this type of " $z$ " is identified with the $s$ - phoneme." 
Swahili pilipili [< Ar. filfil (WeHR 727a) 'pepper'] 'pepper; seeds and pods' > Luganda 'ppiripiri 'pepper'; Madi pilipíli 'hot pepper'; Pokot pilipíl 'pepper';

Swahili -pima v. 'to measure, to weight' > Luena ku-píma 'mesurer'; Madi ìpimà v. 'to measure, to weigh etc.; to examine a patient; to time, to assess';

Swahili pipa [< Port. pípa < Lat. volg. pipa from pipare)] 'cask, barrel, tub'> Acooli piipà 'barrel'; Dholuo pipa 'barrel'; Gikuyu ĩbuba 'barrel, drum'; Kikamba iviva 'drum (oil, petrol)';

Swahili rahisi [<Ar. rakīṣ (WEHR 332b) 'low-priced'] a./v. 'cheap, easy, light (in sense of easy)' > Pokot ràíis a. 'cheap';

Swahili raia [< Ar. raciya (WeHR 346b) 'subjects'] 'a subject, e.g. of the king'> Gikuyu raiya 'citizen, civilian; subject';

Swahili rais, mrais [< Ar. ra '̄̌s (WEHR 318a) 'head; chief; president'] 'president' > Rendille Raáyis 'President';

Swahili ratli, ratili [< Ar. ratl (WeHR 345a) 'rotl, a weight'] '16 wakia, 1 lb.' > Dholuo ratili 'weight'; Gikuyu ratiri 'pound weight; scales, balance'; Madi ràtìlì/rùtūlù 'pounds, the weight';

Swahili risasi, lisasi [< Ar. rașāṣ (WEHR 342b) 'lead; bullets'] 'lead, solder, tin, bullet' $>$ Gikuyu rithathi, rithathi 'bullet; ball-bearing; soldering-iron'; Luganda 'ssasi 'lead; solder; bullet'; Nyakyusa ilisasi 'bullet';

Swahili robo [<Ar. $r u b^{c}$, pl. $\operatorname{arb} \bar{a}^{c}$ (WeHR 322b) 'quarter, fourth part; a dry measure'] 'a fourth part, quarter' $>$ Gikuyu warobo 'one shilling and 50 cents';

Swahili -roga n./v. 'to bewitch'> Madi ìrūgà v. 'to bewitch';

Swahili roho [< Ar. rūh (WEHR 365a) 'breath of life, soul; spirit (in all senses)'] 'soul, spirit, life'> Gikuyu roho 'soul, spirit';

Swahili ruhusa, ruksa [<Ar. rukșa (WEHR 332b) 'permission; authorization; permit'] 'autorisation, permission, liberté, congé'> Dholuo rukca 'a permission, absence from work'; Luganda lùkusà 'permission, leave';

Swahili rushwa [< Ar. rišwa, rušwa, rašwa (WEHR 342a) 'bribe'] 'bribe' > Nyakyusa uluswa 'bribe';

Swahili saa [<Ar. $s \bar{a}^{c} a$ (WEHR 441b) 'while; hour; watch'] 'an hour; time; watch, clock' $>$ Gikuyu thaa 'clock, watch; hour'; Kuria saa -, '- o'clock'; Luganda 'ssaàwa 'hour; watch; clock; time of day'; Nyakyusa isala 'hour, watch, clock'; Pokot sáà 'watch, hour, time';

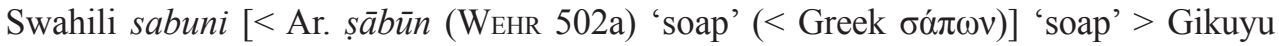
thabuni 'soap; cake of soap'; Luganda 'ssà 'bbuùni 'soap'; Madi sàbú '(short for) soap'; Pokot sàpónìyón 'soap'; Rendille saabbúun 'soap';

Swahili sadaka [< Ar. șadaqa (WEHR 509a) 'alms; charity; prescribed alms tax'] 'a religious offering, sacrifice, alms, act of charity'> Rendille sadákha (?) 'libation (of milk)'; Swahili safari [<Ar. saffara v. (WeHR 412b) (II) 'to send on a journey' (III) 'to travel'] 'a journey, voyage, expedition' > Gikuyu thabari 'expedition with porters, expedition; party bound on an expidition; followers, retainers (e.g. of chief)'; Kuria esabuari 'a safari, journey’; Luganda ‘ssà `ffaàli ‘journey; caravan of porters’; Madi sàfárì 'journey’; 
Swahili safina [< Ar. safina (WeHR 414a) 'ship, vessel, boat'] 'a ship, a vessel, Noah's ark'> Gikuyu thabina 'Noah's ark; ship';

Swahili sahani [< Ar. șạ̣n (WEHR 505a) 'dish; plate; phonograph record'] 'dish, plate' > Gikuyu thani 'plate, saucer'; Luena disahányi 'assiette'; Luganda ‘ssàwaàni, ‘ssaani, 'ssòwaàni 'plate; dish'; Nyakyusa isahani 'plate'; Rendille saháni 'plate';

Swahili sahihi [< Ar. șah̄h (WEHR 503b) 'veritable, real; authentic'] a./v. 'attestation, guarantee, signature' > Gikuyu thahihi 'signature';

Swahili saini $[<$ En.] 'signature' $>$ Dholuo cai 'sign';

Swahili sajini $[<$ En.] 'sergeant' $>$ Dholuo cagin 'sergeant';

Swahili sajini-meja [< En.] 'sergeant-major'> Dholuo cagin meja 'sergeant-major';

Swahili sala [< Ar. șalāh (WEHR 524a) 'the official Islamic prayer ritual'] 'prayer' > Gikuyu thara 'prayer, collect'; Luganda -saàla v.i. 'pray in a set form, pray in Mohammedan way';

Swahili samaki [< Ar. samak (WeHR 431a) 'fish'] 'fish'> Gikuyu thamaki 'fish';

Swahili samli [? < Ar. samm (WeHr 431b) 'clarified butter, cooking butter'] 'ghee, native butter' > Gikuyu thamuri 'ghee, clarified butter';

Swahili sanduku [< Ar. șandūq (WeHR 526a) 'box'] 'chest, box, trunk, case' > Gikuyu ithandũkũ 'box, locker; suit-case'; Lingala sandúku 'caisse, malle, coffre'; Luganda 'ssanduùko 'box; chest'; Madi sàndúù/sàndúkù 'suitcase; box; briefcase; coffin'; Rendille sandúukh 'large box, trunk (made of metal or wood, for storing or transporting goods)';

Swahili sayansi $[<$ En.] 'science' $>$ Dholuo cayanci 'science';

Swahili sementi* [< En.] 'cement'> Rendille simíti 'cement';

Swahili seng'enge 'fencing wire; barbed wire' > Rendille segeénge 'chain link fencing; chain link fence';

Swahili senti $[<$ En.] 'cent' > Dholuo cente 'cents, shilling';

Swahili Septemba [< En.] 'September'> Dholuo Ceptemba 'September';

Swahili serikali [< Pers. ] 'Government, public authority, an official' > Ateso eserikalet 'soldier'; Gikuyu thirikari 'government'; Luganda mùserikalè 'soldier'; Nyakyusa isilikali 'government'; Rendille sirgáal 'government';

Swahili setifiket* [< En.] 'certificate'> Dholuo cabidiket 'certificate, some people now call it catipiket';

Swahili shabaha, shebaha [<Ar. šabah̆, šabḥ (WEHR 451b) 'shape; apparition; phantom'] 'a target, aim; aim (with a weapon), sight (of a gun)'> Gikuyu cabaa 'shooting practice range; good shot, aim (in shooting)'; Luganda 'ssà 'bbaàwa 'target; mark';

Swahili shahidi [<Ar. šāhid (WEHR 489b) 'witness'] 'a witness, a martyr'> Gikuyu caĩri 'witness';

Swahili shati $[<$ En.] 'shirt' > Dholuo cati 'shirt';

Swahili (-)shauri [< Ar. šāwara v. (WeHr 492a) (III) 'to ask s.o.'s advice'] n./v. 'plan; design; advice, counsel' > Gikuyu cauri 'affair, business; discussion, dispute';

Swahili shayiri [< Ar. ša $a^{c} \bar{\imath}$ (WEHR 474a) 'barley'] 'barley'> Gikuyu cairi 'barley'; Luganda è ssàyiri 'barley'; 
Swahili shemasi [<Ar. šammās (WEHR 486a) 'deacon'] 'a deacon'> Gikuyu mũcemathi 'a deacon (of the Anglican Church)';

Swahili shetani [< Ar. šaitān (WEHR 497b) 'Satan, devil'] 'an evil spirit, demon, devil, Satan'> Nyakyusa setano 'Satan, devil';

Swahili shuka [? Ar.] 'sheet'> Acooli cöka 'sheet'; Dholuo cuka 'a sheet of cloth, a bedsheet';

Swahili sidiria [< Ar. șadrīya (KAzIMIRSKI: I, 1319b) 'veste, gilet; chemisette'] 'a cloth worn by some women just below the breasts to support them, also called kanchiri' > Nyakyusa isindilila 'brassiere, bra';

Swahili sigara* [< Ar. sigāra (WEHR 397b) 'cigarette'] 'a cigarette' > Gikuyu thigara 'cigarette'; Rendille sigaára 'cigarette';

Swahili sikukuu 'feast day' > Rendille sukuúku '1. feast, celebration 2. (public) holidays';

Swahili -silimisha [< Ar. aslama (WeHr 425a) v. (IV) 'to become a Muslim'] v. 'make a Muhammadan'> Gikuyu thirimithia v.t. 'convert to Islam';

Swahili simu [< Pers.] 'telegraph, telegraphic message' > Dholuo cim 'telephone, wireless, telegraph, fax, etc.'; Gikuyu thimũ 'telegram, telephone'; Madi st́mù 26 'telephone; telephone message';

Swahili sindano 'a needle' > Luena shindányi 'aiguille';

Swahili sinia [<Ar. șin̄yya 'a large, round metal plate'] 'tray'> Madi sغ̀níà 'tray; roundabout';

Swahili sita [<Ar. sitta (WeHr 397a) 'six'] n./a. 'six'> Gikuyu thita a. 'only used in conjunction with thaa 'clock, watch; hour', q.v.';

Swahili soko [< Ar. sūq (WEHR 443a) 'market'] 'market'> Gikuyu thoko 'market, marketplace'; Kuria esokoni 'a market'; Madi sókò/shu 'market'; Pokot máak't 'market'; Rendille sókko 'market-place';

Swahili soksi [<En.] 'sock'> Dholuo coksi'7 'socks, stockings';

Swahili -soma v. 'to read'> Ateso soma v. tr. 'to read';

Swahili -staarabu [< Ar. ${ }^{C} R B$ V. (WeHR 601a) (II) 'to Arabize; to translate into Arabic' (X) 'to become an Arab'] v./a. 'be wise, be civilized' > Gikuyu ũthitarabu 'civilisation, culture';

Swahili stampu $\left[<\right.$ En.] $>$ Dholuo citam ${ }^{28}$ 'stamp';

Swahili stesheni $[<$ En.] $>$ Dholuo citecen' 29 'station';

Swahili sweta [< En.] 'sweater' > Dholuo cweta ${ }^{30}$ 'sweater';

Swahili sufuria [<Ar. șufr (WeHR 517b) 'brass; money'; șufrīya (Dozy: I, 835b) 'vase de cuivre, chaudron'] 'metal cooking-pot - of copper or iron, sometimes of a very large size'

${ }^{26}$ Blackings marks the word as a loan from Arabic (sic!) in his dictionary.

27 This loan could have arrived directly from English.

${ }^{28}$ This loan could have arrived directly from English.

${ }^{29}$ This loan could have arrived directly from English.

${ }^{30}$ This loan could have arrived directly from English. 
$>$ Acooli cùpùriíà, cibìriíà 'metal cooking pot'; Dholuo cipuria, cibiria 'saucepan, metal cooking pot, usually of alluminium'; Gikuyu thaburia, thuburia 'open metal cookingpot, saucepan'; Rendille subbúrya 'metal cooking pot, sufuria';

Swahili sukari [< Ar. sukkar (WEHR 417b) 'sugar'] 'sugar'> Gikuyu cukari 'sugar'; Lingala sukáli 'sugar; sucré; douceur’; Luganda ‘ssùkaàli 'sugar'; Nyakyusa isúkali 'sugar’; Pokot sùkáarìn 'sugar';

Swahili sumu [< Ar. samm, pl. samūm (WEHR 427b) 'poison'] n./v. 'poison' > Gikuyu thumu '(of snake or other wild animal) poison from a bite';

Swahili sura [< Ar. sūra (WEHR 441a) 'chapter of the Koran'] 'a chapter of a book' > Luganda 'ssuula 'chapter';

Swahili suruali [<Ar. sirwāl (WEHR 408b) 'trousers, pants'] 'trousers'> Acooli còròwáàl 'trousers'; Dholuo curuwal 'a pair of trousers'; Gikuyu thuruarĩ, thuraarĩ 'trousers, knickers'; Luganda 'sseruwalè 'trousers'; Pokot súrwâl 'trousers, shorts'; Madi sùrùwálì 'pair of shorts'; Nyakyusa isúlúbali 'pants';

Swahili taa 'a lamp - of any kind' > Acooli tàwacà 'lamp'; Ateso etala 'lamp'; Gikuyu tawa/taa 'lantern, lamp'; Luganda è 'ttaala 'lantern'; Nyakyusa itala 'lamp'; Pokot táà 'lamp'; Rendille táa 'lamp';

Swahili taji [<Ar. tāj (WEHR 99a) 'crown'] 'a crown, coronet'> Gikuyu tanji 'crown';

Swahili -takatifu a. 'cleansed, clean, pure, holy'> Luganda -tàkàtifu a. 'holy';

Swahili talaka [< Ar. talāq (WEHR 567a) 'divorce'] 'divorce' > Nyakyusa italaka 'divorce';

Swahili tarehe [< Ar. ta'rīk (WEHR 12b) 'history'] 'date, annals, chronicle, journal, history' > Kuria etarehe 'a date, day; (of a woman) menstrual date, periods'; etariki 'date (of the month)';

Swahili theluji [<Ar. talj, pl. tulüj (WeHR 101b) 'snow'] 'snow' > Gikuyu therunji, tharunji 'snow'; Luganda 'sserùji 'snow';

Swahili thenashara [< Ar. itna 'ašara (WEHR 108a) 'twelve'] n./a. 'twelve, for kumi na mbili'> Gikuyu thinacara '6 a.m. or 6 p.m.';

Swahili -thubutu [< Ar. tubūt (WEHR 101b) 'constancy, immutability; certainty'] v. 'have courage to'> Gikuyu thubuticia v. '(of Anglican Church rite) confirm, hold confirmation service (via Swahili tumbutisha)';

Swahili tofali [<Ar. tufāl (WEHR 562b) 'potter's clay; argil; loam'] 'a brick, tile'> Acooli mùtàfalì 'brick'; Gikuyu itubari, iturubarĩ 'brick, cement block';

Swahili tunda, pl. matunda 'a fruit of any kind'> Rendille matuúnda/mutuúnda '(single) fruit, (piece of) fruit';

Swahili ubani [<Ar. lubān (WEHR 856b) 'frankincense'] 'frankincense'> Gikuyu ubani 'frankincense';

Swahili ubao 'a board, plank'> Luena lumbàw 'planche';

Swahili ufunguo '(1) act of opening; (2) a key' > Acooli kìpfuyà 'key';

Swahili uji 'gruel or soup'> Rendille újji 'porridge'; 
Swahili ukurasa [<Ar. kurrāsa (WEHR 820b) 'quire; booklet; notebook, copy book; fascicle (of a book)'] 'sheet or strip of paper, leaf or page of a book'> Gikuyu ũkuratha 'page';

Swahili Ulaya [<Ar. wilāya (WEHR 1100b) 'souveraineté; district administratif dirigé par un waill, wilaya (autrefois, du temps de l'empire ottoman), province'] '(1) a land, esp. if not further defined, Europe (2) a district, in thissewnse it is usually wilaya' > Gikuyu Rũraya, Uraya 'Europe'; Nyakyusa iwilaja 'district';

Swahili upawa 'a ladle made of coconut shell; in gen. (wooden) ladle' > Luena lupàwa 'cuillier';

Swahili upepo 'wind, breeze'> Luena ku-pèpa v. 'souffler';

Swahili ushuru [<Ar. 'ušr, $\mathrm{pl}^{c}$ 'ušūr (WEHR 614a) 'one tenth, tenth part; tithe'] 'taxation, tax, customs duty, rate, rent' > Luganda bùsuùlu 'rent; ground-rent';

Swahili $u z i$ 'thread, cotton, string' > Madi úzi 'thread';

Swahili wakati [<Ar. waqt (WEHR 1087a) 'time'] 'time, season'> Luganda wàkàtî adv. 'in the middle; midway';

Swahili wilaya [<Ar. wilāya (WEHR 1100b) 'sovereignty; administrative district headed by a vali, vilayet (formerly, under the Ottoman Empire), province'] 'parish, district, province'> Gikuyu Rũraya, Uraya 31 'Europe'; Nyakyusa iwilaja 'district';

Swahili wino ${ }^{32}[?<$ Ar.] 'ink'> Luganda bwîno 'ink';

Swahili zabibu [< Ar. zabīb (WEHR 372b) 'raisins'] 'raisin, graipe'> Gikuyu thabibũ 'graipe'; Luganda mùzabbibù 'vine; per ext. grape (via Swahili mzabibu)';

Swahili zaburi [<Ar. zabūr (WeHR 372b) 'Psalms, Psalter'] 'a psalm, the psalter' > Luganda zà 'bbuli 'psalm'; Nyakyusa sabuli ‘psalm';

Swahili zamu [< Ar. zām (KAZIMIRSKI: I, 1029a) 'quart (de toute chose)'] 'properly, a six hours' spell of work, or watching' > Nyakyusa isamú 'turn';

Swahili zeituni [<Ar. zaitūn (WeHr 388a) 'olive tree; olive(s)'] 'olive'> Luganda 'zzeyituùni 'olive';

This list of 268 Swahili words was quite productive in giving loans in a few East African languages. Among them we can see from Table I that the highest stock was formed by Arabic loans, followed by Bantu words and English loans.

Table 1

\begin{tabular}{|c|c|c|c|c|c|c|c|}
\hline Arabic & ? Arabic & Persian & Hindi & Turkish & English & Port. & Bantu \\
\hline 160 & 11 & 4 & 2 & 1 & 26 & 5 & 59 \\
\hline
\end{tabular}

From Table II we can observe that the highest number of loans from Swahili is in Gikuyu, Luganda and Rendille, where the majority of items is concentrated.

31 The word is a loan from another Swahili word, ulaya 'a land, esp. if not further defined, Europe; district', which always comes from the same Arabic wilāya (WEHR 1966: 1100b).

32 In Swahili the word itself is a loan from Arabic win (?) according to Johnson. 
Table 2

\begin{tabular}{|l|c|c|c|c|c|c|c|c|c|}
\hline Languages & $<\mathrm{Ar}$ & $<$ Ar & $<$ Per & $<$ Hindi & $<$ Turk & $<$ En & $<$ Port & $<$ Bantu & Total \\
\hline Acooli & 11 & 2 & 1 & 2 & & 1 & 1 & 9 & 27 \\
\hline Ateso & 1 & 1 & 1 & & & & & 5 & 8 \\
\hline Bari & 2 & & & 1 & & 1 & & 1 & 5 \\
\hline Dholuo & 16 & 2 & 2 & 1 & & 19 & 2 & 5 & 47 \\
\hline Gikuyu & 106 & 4 & 2 & & & & 2 & 5 & 119 \\
\hline Lingala & 7 & 1 & & & & & & & 8 \\
\hline Luena & 4 & & & & & 2 & & 11 & 17 \\
\hline Luganda & 58 & 3 & 1 & & & & 4 & 8 & 74 \\
\hline Madi & 23 & & 1 & 2 & 1 & 3 & 1 & 6 & 37 \\
\hline Nyakyusa & 28 & & 1 & & 1 & & 1 & 2 & 33 \\
\hline Pokot & 18 & & & & & & 1 & 3 & 22 \\
\hline Rendille & 27 & 2 & 2 & & & 2 & & 23 & 56 \\
\hline Shona & 3 & & & & & & & & 3 \\
\hline Xhosa & 1 & & & & & & & & 1 \\
\hline
\end{tabular}

At last, if we analyze the material collected from a grammatical point of view, we can observe that most of Swahili loanwords are nouns. The verbs are very few: -badilisha; $-f i$ kiri; -funga; -koroga; -pima; -roga; -silimisha; -soma; -thubutu and two of them -fikiri and -koroga are no more verbs respectively in Gikuyu and Rendille.

As it regards the other aspects of grammar we have really few adjectives, inter alia, only the first two are still considered adjectives in the languages lent: -geni; -takatifu; chafu; -faransa; bure; rahisi; sahihi; laghai; and one item, respectively, as an adverb, labda, as a conjunction, basi, and as an interjection, hayy.

\section{REFERENCES}

BALDI Sergio. 2008. Dictionnaire des emprunts arabes dans les langues de l'Afrique de l'Ouest et en swahili. Paris: Karthala.

AwDE Nicholas. 2000. Swahili-English; English-Swahili Dictionary (Hippocrene Practical Dictionary). New York:

Hippocrene Books.

BAKHRESSA Salim K. 1992. Kamusi ya Maana na Matumizi [(Swahili) Dictionary of definition and usage]. Nairobi-

Dar es Salaam-Kampala: Oxford University Press.

Barlow A. Ruffell, Benson T.G. 1975. English-Kikuyu Dictionary. Oxford: Clarendon Press.

Benson T.G. 1964. Kikuyu-English Dictionay. Oxford. At The Clarendon Press.

Blackings Mairi John. 2000. Ma'di English - English Ma'di Dictionay (Languages of the World/Dictionaries 25).

Muenchen: Lincom Europa.

Boucneau Jacques. 1987. A Tentative Linguistic Bibliography of Swahili 1964-1984: Basics, Phonology \& Morphophonology, Morphology, Syntax, Lexicography (Working Papers in Kiswahili No. 2). Rijksuniversiteit te Gent. Seminarie voor Swahili en de Taalproblematiek van de Ontwikkelingsgebieden; cf. Sergio Baldi, AION 1991, 51, 2: 185-213.

Crazzolara Josef Pascal. 1955². A Study of the Acooli Language. Grammar and Vocabulary. London: Oxford University Press for the International Institute of African Languages \& Cultures.

Crazzolara Josef Pascal. 1978. A Study of the Pokot (Suk) Language. Grammar and Vocabulary. Bologna: Editrice Missionaria Italiana.

Dale Desmond. 1987. A Basic English-Shona Dictionary. Gweru (Zimbabwe): Mambo Press.

Dozy Reinhart. 1881. Supplément aux dictionnaires arabes. T. 1-2. Leyde: Brill. 
FelBerg Knut. 1996. Nyakyusa-English-Swahili and English-Nyakyusa Dictionary. Dar es Salaam: Mkuti na Nyota Publishers Limited.

Finlayson Rosalie (ed.). 1999. African Mosaic (Festschrift for J.A. Mosaic). Pretoria: Unisa Press, University of South Africa.

FreYTAG Georg Wilhelm. 1830-1835. Lexicon arabico-latinum. Halis Saxonum. C.A. Schwetschke et Filium.

Ghaleb Edouard. 1966. Dictionnaire des sciences de la nature. T. 1-3. Beyrouth: Édition de l'imprimerie catholique.

Gorman T.P. (ed.). 1972. A Glossary in English, Kiswahili, Kikuyu and Dholuo. London: Cassell \& Company.

Hannan M. 1968. Standard Shona Dictionary. London: MacMillan \& Co.

Institute of Kiswahili Research. 1996. Tuki English-Swahili Dictionary. Kamusi ya Kiingereza-Kiswahili. Dar es Salaam: University of Dar es Salaam.

JoHnson Frederick. 1939. A Standard Swahili-English Dictionary. London-Oxford: O.U.P.

KazimiRski A. de Biberstein. 1860. Dictionnaire arabe-français. T. 1-2. Paris: Maisonneuve et Cie.

Kitching A.L. 1915. A Handbook of the Ateso Language. London: Society for Promoting Christian Knowledge.

KNAPPERT Jan. 1972-1973. "The Study of Loan Words in African Languages." Afrika und Übersee 56(4): 283-308.

KNAPPERT Jan. 1999. "Loanwords in African Languages.” In: FinLAYSON 1999: 203-220.

Mioni Alberto. 1967. "La bibliographie de la langue swahili. Remarques et supplément à la Swahili Bibliography de M. Van Spaandonck". Cahiers d'Études Africaines 27(7), 485-532.

Muniko S.M., Muita oMagige B., Ruel M.J. (eds.). 1996. Kuria-English Dictionary (Monographs from the International African Institute). Hamburg: Lit Verlag.

Muratori C. 1948. English Bari-Lotuxo-Acoli Vocabulary. Okaru: Catholic Mission Printing Press.

MuRPHY John D. 1972. Luganda-English dictionary (Publications in the languages of Africa, 2). Washington D.C.: Consortium Press, The Catholic University of America Press.

Nicolas Francis. 1953. La Langue berbère de Mauritanie (Mémoires de l'Institut français d'Afrique noire, n 33). Dakar: Ifan.

Odonga Alexander. 2005. Lwo-English Dictionary. Kampala: Fountain Publishers.

Rechenbach Charles W. 1967. Swahili-English Dictionary. Washington: The Catholic University of America Press.

Sacleux Charles. 1939. Dictionnaire swahili-français. Paris: Institut d'Ethnologie.

Snoxall R.A. (ed.). 1967. Luganda-English Dictionary. Oxford: Clarendon Press.

SPAANDONCK Marcel van. 1965. Practical and Systematical Swahili Bibliography: Linguistics 1850-1963. Leiden: Brill.

WeHr Hans. 1966. A Dictionary of Modern Written Arabic. Ed. by J. Milton Cowan. Wiesbaden-London: Otto Harrassowitz. 